\title{
IMPROVEMENT OF BIOPHYSICAL MICROMIXER DESIGN
}

\author{
Chin-Tsan Wang \\ Department of Mechanical and Electro-Mechanical Engineering, National I-Lan University, I lan, Taiwan., \\ ctwang@niu.edu.tw \\ Tzu-Yang Hu \\ Department of Mechanical and Electro-Mechanical Engineering, National I-Lan University, I lan, Taiwan.
}

Follow this and additional works at: https://jmstt.ntou.edu.tw/journal

Part of the Engineering Commons

\section{Recommended Citation}

Wang, Chin-Tsan and Hu, Tzu-Yang (2011) "IMPROVEMENT OF BIOPHYSICAL MICROMIXER DESIGN," Journal of Marine Science and Technology: Vol. 19: Iss. 4, Article 12.

DOI: $10.51400 / 2709-6998.2184$

Available at: https://jmstt.ntou.edu.tw/journal/vol19/iss4/12

This Research Article is brought to you for free and open access by Journal of Marine Science and Technology. It has been accepted for inclusion in Journal of Marine Science and Technology by an authorized editor of Journal of Marine Science and Technology. 


\section{IMPROVEMENT OF BIOPHYSICAL MICROMIXER DESIGN}

Acknowledgements

The authors would like to acknowledge the kind funding support from NSC Taiwan under contract \#NSC-98-2221-E197-018. 


\title{
IMPROVEMENT OF BIOPHYSICAL MICROMIXER DESIGN
}

\author{
Chin-Tsan Wang* and Tzu-Yang Hu*
}

Key words: biophysical micromixer, mixing coefficient, passive micromixer.

\begin{abstract}
In a passive micro-scale mixer, the complete mixing of two or more fluids within a reasonable time period plays an important role. Microfluidic transportation and effective mixing are of importance and require examination. The biophysical micromixer addressed by authors would be utilized because of its excellent ability to enlarge the interface and reduce the diffusion length during the mixing process. Here, the mixing efficiency, pressure drop and Aspect ratio (AR) at an optimal inlet Reynolds ratio will be studied. Some useful results will be addressed: First, the dimensions of $A_{X}, G_{X}, G_{Y}, F_{X}, H_{X}, H_{Y}$ and $\mathrm{J}$ would be effective factors when mixing the biophysical micromixer. Second, the recirculation region would play an important role in the mixing with an increment of $33.03 \%$ with respect to the original prototype because it would escalate the two-flow interaction. Lastly, the side wall effect will influence both the mixing performance and the pressure drop. An aspect ratio of $\mathrm{AR}=10$ will be suggested because it exhibits the highest mixing coefficient of 0.907 and the lowest pressure drop than previous studies cases. These findings will show the greater feasibility of the biophysical micromixer being mixed in a limited space. Additionally, these results would be useful for the mixing improvement of passive micromixer.
\end{abstract}

\section{INTRODUCTION}

Rapid mixing is crucial to microfluid systems, which are now widely used in biochemistry analysis, chemical synthesis, drug delivery, high-throughput screening, among others [7, 26]. In microsystems, their small size prohibits turbulent mixing. The Reynolds figure is generally less than 100, and can be as low as 0.01 , which lies within the creeping regime. Hence, the mixing in microchannels is predominantly governed by mo-

Paper submitted 02/05/10; revised 06/05/10; accepted 06/10/10. Author for correspondence: Chin-Tsan Wang (e-mail: ctwang@niu.edu.tw).

*Department of Mechanical and Electro-Mechanical Engineering, National I-Lan University, I lan, Taiwan. lecular diffusion. If the thickness of the lamella structure of the fluid is much larger than the characteristic diffusion length, it is difficult to obtain rapid mixing.

To enhance fluid mixing, either active or passive methods can be employed. Different mixing principles and micromixer designs have been reported in order to show improved fluid mixing within the microchannel. Micromixers can mainly be categorized as either active micromixers or passive micromixers. An active micromixer requires an external power source to facilitate mixing. These external energy supply points cause a periodic variation of flow rates, microimpellers, ultrasonic effects, and so on $[8,22,32]$. The structures are often complicated and require a complex fabrication process as a transmission mechanism for the external energy source and a mixing chamber is needed. Although an active micromixer's mixing time and microchannel length required for uniform mixing is less than that for a passive micromixer, the fact that it requires external power makes it difficult for it to be integrated with other microfluidic devices. The relatively higher power consumption and cost make active mixers less attractive for use as disposable applications. Contrarily, passive micromixers, including the biophysical micromixer addressed in this study, are simple to operate because the mixing process occurs along with a structural change of the microchannel, making them both attractive and suitable for integration with other devices. A passive micromixer, however, acts upon the pressure drop of the fluids.

The construction of a passive micromixer is simpler and its operation is less complicated than that of an active micromixer. In this study, various mixing principles were applied to the passive micromixers to achieve higher efficiencies of mixing. These included interdigital multilamination, split and recombination, diffusion length decrease, vortex generation, and chaotic mixing [2-6, 10, 12-14, 17, 20-25, 31], to name just a few. An interdigital micromixer with alternating feed channels that periodically created liquid multilamination was addressed and geometrics were used focusing on the reduction of the lamellae width and the acceleration of mixing $[9,13,19]$. In addition, a split and recombination mixer addressed by Lee et al. [15] increased the contact surface area exponentially. Lin et al. [16] used a circular mixing chamber to generate a vortex and two inlet channels which were then divided into eight individual channels. Stroock et al. [24] demonstrated that a staggered herringbone structure generates chaotic mix- 
ing. Two key issues in micromixer development usually concern a simple system design with a high mixing efficiency in addition to effective techniques for examining mixing efficiency $[18,30]$. It is known that the flow inside microchannels is predominantly laminar and the Reynolds numbers are usually lower than 10 . Therefore, the mixing of fluids in microchannels is not easily implemented via mechanical stirring methods because of size limitations and fabrication difficulties [1]. Improvement in the flexibility and performance of microfluidic systems by incorporating a number of processes, including fluid handling and fluid motion, that cause rapid mixing on a micro scale, can become a challenging problem [20].

In general, most traditional passive micro-mixers have been constructed with straight fluid channels and designed with a combination of fillisters and/or fold paths to enhance the mixing effect [29]. However, the design of a straight channel requires a longer length by which to achieve the goal of uniform mixing. Therefore, it is always associated with the problems of mixer sizes and full-field inspections. To achieve a better mixing effect, an efficient passive micro-mixer usually involves complex 3-dimensional geometries which are utilized to improve fluid lamination, stretching and folding. But that would cause a substantial pressure drop and present difficulties in the design and fabrication process. In order to overcome these problems, a novel passive micro-mixer, which utilizes a biophysical concept and possesses a higher flow uniformity and a lower pressure drop, is addressed by the authors $[27,28]$. In this study, an impinging channel and recirculation chamber will be added in the prototype of biophysical micromixer to enlarge the interface and reduce the diffusion length. The finding of an optimal design for a biophysical mixer that possesses an exceptionally high mixing performance will also be presented. This will be shown to be the highest that has ever been attained in any previous case studies.

\section{NUMERICAL METHOD}

In this study, an optimal design that originated from the prototype of a biophysical micromixer, whose biophysical-type micro-channel was created to mimic the features of vascular flow networks as shown in the Fig. 1, was investigated $[27,28]$. In this case, the fluid in the middle channel, labeled as Fluid $\mathrm{I}_{2}$, is assumed to contain a species such as protein or DNA, and the dimensionless concentration is set to unity for the Inlet 1 channel. In addition, the fluids in channels $I_{1}$ and $I_{3}$ are all the same and the concentration are assumed to be zero in the name of another fluid. The equations that govern the mixing process can be obtained by solving the continuity equation in the form of (1), momentum equation shown in (2), and diffusion equations defined in the format of (3):

$$
\begin{gathered}
\nabla \bullet \vec{V}=0 \\
\frac{\partial \vec{V}}{\partial t}+\vec{V} \bullet \nabla \vec{V}=-\nabla p+\frac{1}{\operatorname{Re}} \nabla^{2} \vec{V}
\end{gathered}
$$

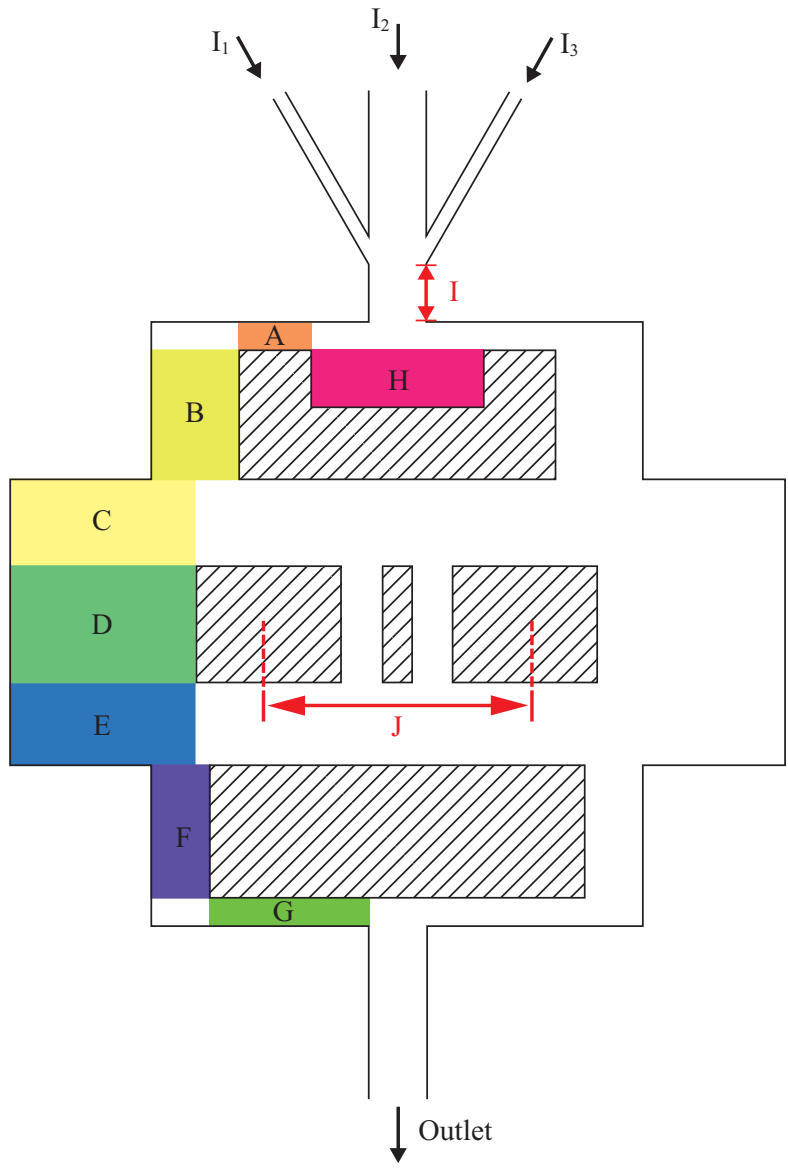

Fig. 1. Dimensional factors of biophysical micromixer.

$$
\frac{\partial C_{i}}{\partial t}+\vec{V} \bullet \nabla C_{i}=\frac{1}{\operatorname{Re} S c} \nabla^{2} C_{i}
$$

Here, Re is the Reynolds number and is defined as $\mathrm{Re}=$ $\frac{\rho V_{0} W}{\mu} ; \mathrm{Sc}$ is defined as $S_{c}=\frac{\mu}{\rho D_{i j}}$ and is the Schmidt number to represent the ratio of the viscosity effect to the diffusion effect. $\mathrm{W}$ is the width of the outlet channel, $\vec{V}$ is the velocity vector, $t$ is time, $p$ denotes pressure, $C_{i}$ represents mole concentration, $V_{0}$ is the characteristic velocity, $\mu$ is the fluid viscosity, $\rho$ is the density of fluid, and $D_{i j}$ is the mass diffusivity. In addition, $P e$ is defined as $P e=\frac{\bar{U} D}{D_{A B}}$ and is the Pelect number to show the ratio of the convection effect to the diffusion effect and will be investigated.

The mixing performance was numerically simulated using a commercial CFD software package, CFD-ACE + and a multiphysics package based on the Finite-Volume method was applied. The program was run on a $2.4 \mathrm{GHz}$ Pentium IV processor with $1 \mathrm{~GB}$ of RAM memory. Mesh-independent tests were performed before the studies took place. An upwind method for solving the multi-block unstructured grid of $2 \times 10^{4}$ 
cells was used as the $2 \mathrm{D}$ computational domain inside the micro-mixer. In addition, the grids ranging from $1.46 \times 10^{5}$ to $9.17 \times 10^{5}$ cells were used in $3 \mathrm{D}$ simulation for an aspect ratio from $\mathrm{AR}=0.5$ to $\mathrm{AR}=10$. The convergent criterion was assumed to be $\pm 10^{-18}$ for the residual of the discrete governing equations in the simulation. The boundary conditions were set at the optimal condition which exhibits outstanding mixing performance at Reynolds number ratio $\operatorname{Rer}=0.85$, defined as $\mathrm{Re}_{r}=\frac{\mathrm{Re}_{1}+\mathrm{Re}_{3}}{\mathrm{Re}_{2}}$ and which had been addressed by a previous study. The resulting flow mixing mechanism would be mainly from the flow convection in the biophysical micro-channels.

In the simulation process, constant inlet velocities whose values are $7.5813 \times 10^{-2} \mathrm{~m} / \mathrm{sec}$ and $4.4864 \times 10^{-2} \mathrm{~m} / \mathrm{sec}$ for channel $I_{1}\left(I_{3}\right)$ and $I_{2}$ respectively set at a given Reynolds number $\operatorname{Re}_{1}=\operatorname{Re}_{3}=0.425$ and $\operatorname{Re}_{2}=1$ as related to the optimal Reynolds ratio Rer $=0.85$ were used for steady-state analysis [27, 28], and the outlet reference pressure was set as zero as the gage pressure. Otherwise, the flow velocity, reference pressure, and concentration were all set to zero as the initial conditions. Although the valve flow was laminar, a rather fine mesh was needed to account for the detailed features of the sorting mechanism. The time limit for each run spanned from two up to three hours.

In this study, a biophysical-type micro-channel, as shown in Fig. 1, was used as a prototype passive micro-mixer. This is because it would not damage a bio-sample in transmission and is easy to fabricate $[27,28]$. The width of the middle channel, labeled as Fluid $\mathrm{I}_{2}$, was set at $20 \mu \mathrm{m}$ and the two identical side entrances, channels $I_{1}$ and $I_{3}$, were set at $7.1 \mu \mathrm{m}$. The outlet channel width $W$ was set at $20 \mu \mathrm{m}$. Although increasing the mixing length could increase the mixing performance for a passive micro-mixer, the total length of the micro-channel was restricted to $370 \mu \mathrm{m}$. This is less than that of other known passive micro-mixers, and was done on purpose for this study because the effect of the mixing length is not the main objective in this experiment. Instead, this study focuses on finding an optimal biophysical micro-mixer which possesses the highest mixing performance of all previous case studies.

Here a mixing coefficient $\varepsilon_{\text {mixing }}$ defined in (4) was employed to find the optimal dimensional structure of a biophysical micro-mixer.

$$
\varepsilon_{\text {mixing }}=1-\frac{1}{W} \int_{0}^{W}\left|\frac{X_{A_{\text {xoulte }}}-0.5}{X_{A_{\max }}-0.5}\right| d x
$$

Here, $X_{A_{\max }}$ is the maximum mole fraction of Fluid A(Fluid $\mathrm{I}_{2}$ ) with the value at unity, $X_{A_{x \text { xoutce }}}$ is the mole fraction of Fluid A at outlet location, and $W=20 \mu \mathrm{m}$ denotes the outlet width of the channel. As $\varepsilon_{\text {mixing }}$ approaches 1, the mixing efficiency approaches a maximum. A larger value of $\varepsilon_{\text {mixing }}$ corresponds to a better mixing performance.

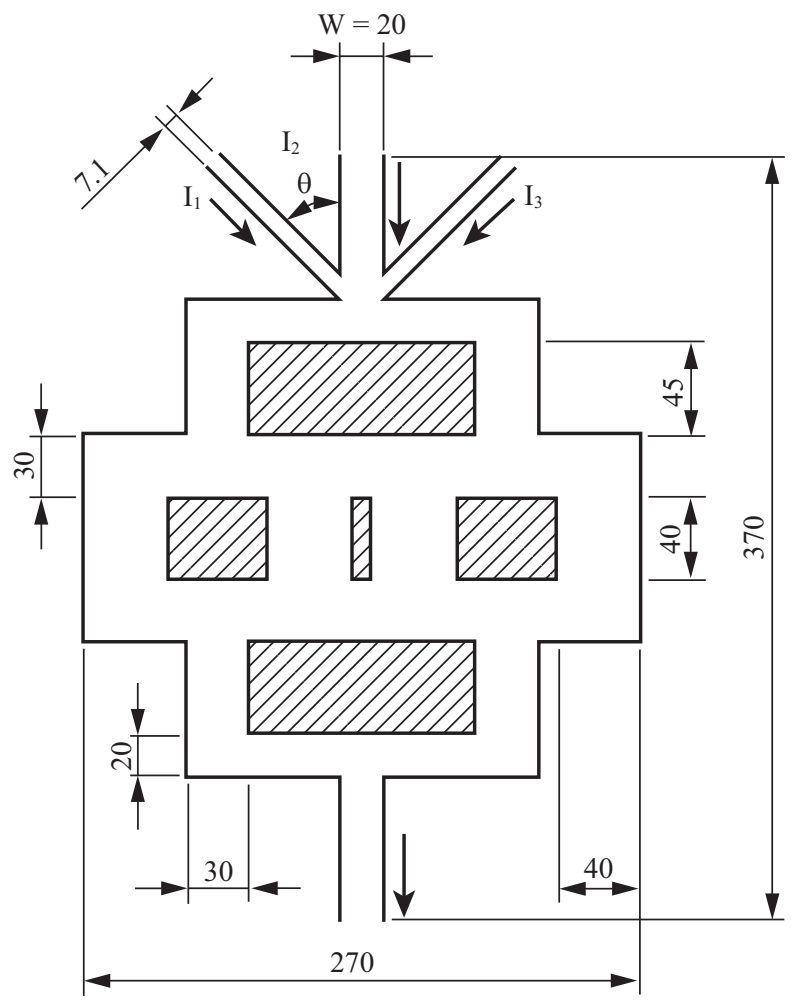

Fig. 2. The original prototype of biophysical micromixer (unit: $\mu \mathrm{m}$ ), the inlet angle of $\theta$ is $30^{\circ}$ and the arrow indicates the inlet and outlet flow direction.

\section{DISCUSSIONS AND RESULTS}

In this study, Fig. 1 shows that an optimal dimensional structure for a micromixer originating from the prototype of Fig. 2 was investigated at Rer $=0.85$. Here, it should be noted that the recirculation region $\mathrm{H}$ and impinging height $\mathrm{I}$ were added because they are important factors in the mixing process.

In addition, dimensions like channel region color, marked as $\mathrm{A}$ to $\mathrm{G}$, and the distance between the two square obstructions at the middle of the micromixer J are shown in Fig. 2. To simplify the dimensional analysis of the micromixer system, a dimension $\mathrm{R}_{\mathrm{X}, \mathrm{Y}}$, where $\mathrm{R}$ is used to name the channel region $\mathrm{A}$ to $\mathrm{G}$, and where the suffix $\mathrm{X}$ and $\mathrm{Y}$ indicate the parallel width and perpendicular height respectively for the different channel regions were applied in this study.

For an optimal analysis of the biophysical micromixer, the value of suffix $\mathrm{X}$ and $\mathrm{Y}$ based on the original dimensions of the micromixer were adjusted in \pm 5 and shown in Table 1. Here, it should be noted that one parameter selected first would be analyzed for each process in order to find the effective factors in the mixing. From a series of analytical procedures, the results showed that the parameters $A_{X}, G_{X, Y}, F_{X}, H_{X, Y}, J$ and I would be effective factors in the mixing. As for the integrated mixing performance of system, the Taguchi method [31, 32] used to determine the coupling effect of parameters was executed in the range of the values shown in Table 2. 
Table 1. Variations of dimension based on the original prototype.

\begin{tabular}{|c|c|c|}
\hline Original dimensions & Increment of suffix & Decrement of suffix \\
\hline $\mathrm{A}_{45,20}$ & $\mathrm{~A}_{50,20} \cdot \mathrm{A}_{45,25}$ & $\mathrm{~A}_{40,20} \backslash \mathrm{A}_{45,15}$ \\
\hline $\mathrm{B}_{30,45}$ & $\mathrm{~B}_{35,45} \cdot \mathrm{B}_{30,50}$ & $\mathrm{~B}_{25,45} \cdot \mathrm{B}_{30,40}$ \\
\hline $\mathrm{C}_{50,30}$ & $\mathrm{C}_{55,30} \cdot \mathrm{C}_{50,35}$ & $\mathrm{C}_{45,30} \cdot \mathrm{C}_{50,25}$ \\
\hline $\mathrm{D}_{40,40}$ & $\mathrm{D}_{45,40} \cdot \mathrm{D}_{40,45}$ & $\mathrm{D}_{35,40} \cdot \mathrm{D}_{40,35}$ \\
\hline $\mathrm{E}_{50,30}$ & $\mathrm{E}_{50,35}$ & $\mathrm{E}_{50,25}$ \\
\hline $\mathrm{F}_{30,45}$ & $F_{35,45} \backslash F_{30,50}$ & $\mathrm{~F}_{25,45} \backslash \mathrm{F}_{30,40}$ \\
\hline $\mathrm{G}_{45,20}$ & $\mathrm{G}_{50,20} \cdot \mathrm{G}_{45,25}$ & $\mathrm{G}_{40,20} \backslash \mathrm{G}_{45,15}$ \\
\hline $\mathrm{J}_{130}$ & $\mathrm{~J}_{138} 、 \mathrm{~J}_{146} 、 \mathrm{~J}_{156}$ & $\mathrm{~J}_{106} 、 \mathrm{~J}_{114} 、 \mathrm{~J}_{122}$ \\
\hline $\mathrm{H}_{0,0}$ & $\mathrm{H}_{60,20}$ & \\
\hline I & $4 \cdot 8 \cdot 12$ & \\
\hline
\end{tabular}

Table 2. Dimensions of effective parameter for biophysical micromixer.

\begin{tabular}{|c|c|}
\hline Dimensions of effective parameters & Variation of suffix \\
\hline$A_{X}$ & $X=10-20$ \\
\hline$G_{X}$ & $X=10-20$ \\
\hline$G_{Y}$ & $Y=45-55$ \\
\hline$F_{X}$ & $X=20-40$ \\
\hline$H_{X}$ & $X=5-20$ \\
\hline$H_{Y}$ & $Y=20-60$ \\
\hline$J$ & $90-170$ \\
\hline
\end{tabular}

Table 3. Optimal dimensions of effective parameter for biophysical micromixer.

\begin{tabular}{|c|c|}
\hline Dimensions of effective parameters & Variation of suffix \\
\hline$A_{X}$ & $X=10$ \\
\hline$G_{X}$ & $X=10$ \\
\hline$G_{Y}$ & $Y=55$ \\
\hline$F_{X}$ & $X=20$ \\
\hline$H_{X}$ & $X=20$ \\
\hline$H_{Y}$ & $Y=20$ \\
\hline$J$ & 90 \\
\hline$I$ & 20 \\
\hline
\end{tabular}

An optimal parameter group possessing a better mixing index of $\varepsilon_{\text {mixing }}=0.8975$ was found and shown in Table 3 . The mixing efficiency of the optimal dimensions would be increased with an increment of $33.02 \%$ based on the original prototype. The main reason, with respect to improving the mixing performance, can be found clearly in Table 4 . The results in Table 4 show the mixing at different downstream locations from the inlet. The recirculation region would play an important role in mixing because it increased the two-flow interaction. Generally speaking, passive micromixers do not
Table 4. The mixing efficiency versus different distance to inlet.

\begin{tabular}{|c|c|c|c|c|}
\hline Distance to inlet & $\mathrm{X}_{80}$ & $\mathrm{X}_{130}$ & $\mathrm{X}_{200}$ & $\mathrm{X}_{370}$ \\
\hline$\varepsilon_{\mathrm{X}}$ & 0.381 & 0.662 & 0.691 & 0.897 \\
\hline
\end{tabular}

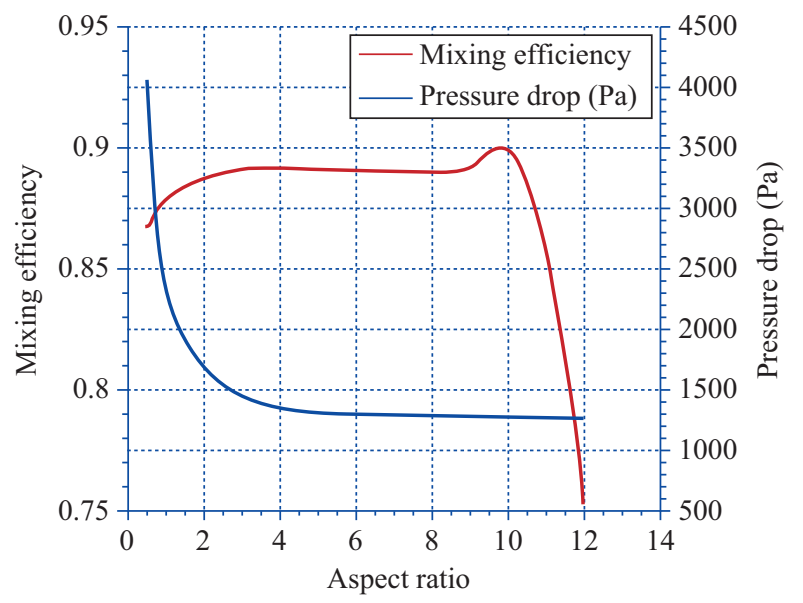

Fig. 3. Aspect ratio, AR, versus mixing efficiency and pressure drop (Pa).

mix well and increasing the mixing length would be a common method used for improvement. However, this result shows the feasibility that this biophysical micromixer could possess a better mixing performance in a limited space of $370 \mu \mathrm{m}$. To simulate the real operation of the mixer, an aspect ratio denoted as $\mathrm{AR}$ and defined in the form of $\mathrm{AR}=\mathrm{D} / \mathrm{W}$ was used to determine the effect of the aspect ratio to mixing and pressure drop at the optimal dimensions of the bio-micromixer, where $\mathrm{D}$ is depth of channel and $\mathrm{W}$ indicates the width of outlet channel. Fig. 3 shows the mixing efficiency and pressure drop at $\mathrm{AR}=0.5$ to 12 at $\mathrm{Rer}=0.85$.

According the new paper [11], Fig. 5 shown in the paper [11] indicates that the mixing index at the exit will approach the limiting value of about 0.9 for the three kinds of flow structure studied at Reynolds number $\mathrm{Re}=267$. Conversely, the pressure drop increases rapidly with the Reynolds number in all studied channels whose flow structure type is a square wave channel, Zig-zag channel and curve channel, respectively. Fig. 8 of paper [11] shows that the value of the pressure drop will be larger than $3.5 \times 10^{4}$ pa for $\operatorname{Re}>267$. This evidence shows that the maximum pressure drop for the biophysical micro-mixer is about 3500 pa when the value is less than one for present results [11] and corresponds to the same mixing index whose value is about 0.9 . In addition, the mixing length of the paper [11] is larger than $2 \mathrm{~mm}$ when it approaches the mixing index of 0.9 , but $370 \mu \mathrm{m}$ will be needed at $\mathrm{Re}_{2}=1$ for the biophysical micro-mixer. These results show that the biophysical micro-mixer has a better mixing performance than that of other passive micro-mixers.

In addition, the results shown in Fig. 3 are addressed as follows: First, the mixing efficiency will increase roughly with 


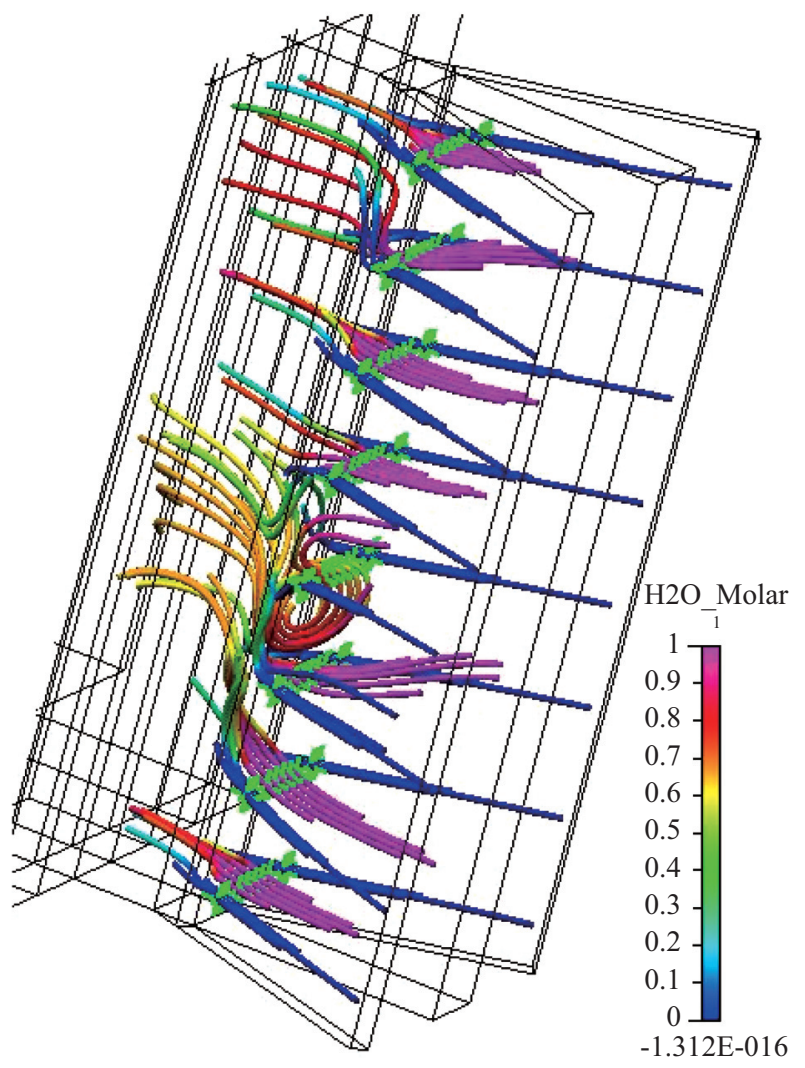

Fig. 4. Flow image of side view for three-inlet intersection section.

AR but decreases once AR $>10$. The mixing efficiency arrives at a maximum value of 0.907 for $A R=10$ because the swirling and asymmetric flow shown in Fig. 4 occur. Conversely, the pressure drop would decrease with AR. Thus, the case of $A R=10$ would be suggested as the optimal design for a biophysical micromixer. The mixing efficiency of the optimal type is increased by about $33.03 \%$ compared to the prototype of the bio-micromixer. These findings would be useful for further experimental confirmation of a biophysical micromixer in the future.

\section{CONCLUSION}

An optimal design for a passive micromixer originating from the biophysical prototype was designed in this study due to its simple shape. In addition, the design possesses a higher mixing performance and lower pressure drop. Some numerical results executed at an optimal flow condition using Reynolds number ratio Rer $=0.85$ were obtained and are addressed as follows:

First, the dimensions of $A_{X}, G_{X}, G_{Y}, F_{X}, H_{X}, H_{Y}$ and $J$ were found to be effective factors in mixing for the biophysical micromixer. Second, the recirculation region plays an important role in mixing with an increment of $33.03 \%$ with respect to the original prototype because it increases the two flow interaction. Finally, the side wall effect will influence the mixing performance and pressure drop. An aspect ratio of $\mathrm{AR}=10$ would be suggested for use as it exhibits the highest mixing coefficient of 0.907 and the lowest pressure drop of all other studied cases. These findings show the feasibility of better mixing within a limited space for the biophysical micromixer. In addition, these results would be beneficial to future experiments.

\section{ACKNOWLEDGMENTS}

The authors would like to acknowledge the kind funding support from NSC Taiwan under contract \#NSC-98-2221-E197-018.

\section{REFERENCES}

1. Aubin, J., Fletcher, D. F., and Xuereb, C., "Design of micromixers using CFD modeling," Chemical Engineering Science, Vol. 60, pp. 2503-2516 (2005).

2. Bessoth, F. G., de Mello, A. J., and Manz, A., "Microstructure for efficient continuous flow mixing," Analytical Communications, Vol. 36, pp. 213-215 (1999).

3. Bhagat, A. A. S., Peterson, E. T. K., and Papautsky, I., "A passive planar micromixer with obstructions for mixing at low reynolds numbers," Journal of Micromechanics and Microengineering, Vol. 17, pp. 10171024 (2007).

4. Branebjerg, J., Gravesen, P., Krog, J. P., and Nielsen, C. R., "Fast mixing by lamination," Proceedings of the 9th Annual International Workshop on Micro Electro Mechanical Systems. San Diego, CA, pp. 441-446 (1996).

5. Ducreé, J., Brenner, T., Haeberle, S., Glatzel, T., and Zengerle, R., "Multilamination of flows in planar networks of rotating microchannels," $\mathrm{Mi}$ crofluidics and Nanofluidics, Vol. 2, pp. 78-84 (2006).

6 Ehrefeld, W., Golbig, K., Hessel, V., Löwe, H., and Richter, T., "Characterization of mixing in micromixer by a test reaction: single mixing units and mixer arrays," Industrial and Engineering Chemistry Research, Vol. 38, pp. 1075-1082 (1999).

7. Giordano, N. and Cheng, J. T., "Microfluid mechanics: progress and opportunities," Journal of Physics: Condensed Matter, Vol. 13, pp. 271-295 (2001).

8. Glasgow, I. and Aubry, N., "Enhancement of microfluidic mixing using time pulsing," Lab on a Chip, Vol. 3, pp. 114-120 (2003).

9. Hessel, V., Hardt, S., Löwe, H., and Schönfeld, F., "Laminar mixing in different interdigital micromixers: I. experimental characterization," AIChE Journal, Vol. 49, pp. 566-577 (2003).

10. Hessel, V. and Löwe, H. "Microchemical engineering: components, plant concepts user acceptance part I," Chemical Engineering and Technology, Vol. 26, pp. 13-24 (2003).

11. Hossain, S., Ansari, M. A., and Kim, K. Y., "Analysis of mixing and flow structure in different passive micro-mixers," Proceedings of the ASME 2009 7th International Conference on Nanochannels, Microchannels and Minichannels ICNMM2009-82116, June 22-24, Pohang, South Korea (2009).

12. Kim, D. S., Lee, S. W., Kwon, T. H., and Lee, S. S., "A barrier embedded chaotic micromixer," Journal of Micromechanics and Microengineering, Vol. 14, pp. 798-805 (2004).

13. Lee, J. and Kwon, S., "Mixing efficiency of a multilamination micromixer with consecutive recirculation zones," Chemical Engineering Science, Vol. 64, pp. 1223-1231 (2009).

14. Lee, S. H., Kang, H. J., and Choi, B., "A study on the novel micromixer with chaotic flows," Microsystem Technologies, Vol. 15, pp. 269-277 (2009).

15. Lee, S. W., Kim, D. S., Lee, S. S., and Kwon, T. H., "A split and recombination micromixer fabricated in a PDMS three-dimensional structure," 
Journal of Micromechanics and Microengineering, Vol. 16, pp. 10671072 (2006).

16. Lin, C. H., Tsai, C. H., and Fu, L. M., "A rapid three dimensional vortex micromixer utilizing self-rotation effects under low Reynolds Number conditions," Journal of Micromechanics and Microengineering, Vol. 15, pp. 935-943 (2005).

17. Liu, R. H., Stremler, M. A., Sharp, K. V., Olson, M. G., Santiago, J. G., and Adrian, R. J., "Passive mixing in a three-dimensional serpentine microchannel," Journal of Microelectromechanical Systems, Vol. 9, pp. 190-197 (2000).

18. Liu, Y. Z., Kim, B. J., and Sung, H. J., "Two-fluid mixing in a microchannel," International Journal of Heat and Fluid Flow, Vol. 25, pp. 986995 (2004).

19. Löb, P., Pennemann, H., Hessel, V., and Men, Y., "Impact of fluid path geometry and operating parameters on 1/1- dispersion in interdigital micromixers," Chemical Engineering Science, Vol. 61, pp. 2959-2967 (2006).

20. Paul, C., Ridha, B. M., and Pierre, S., "A study of passive microfluidic mixers," University of Toronto: Toronto, icmens, pp. 287-293 (2004)

21. Rao, R. S., Prakasham, R. S., Prasad, K. K. S., Rajesham, P., Sarma, N., and Rao, L. V., "Xylitol production by Candida sp.: parameter optimization using Taguchi approach," Process Biochemistry, Vol. 39, pp. 951-956 (2004).

22. Ravella, S. R., Kumar, C. G., Prakasham, R. S., and Hobbs, P. J., "The Taguchi methodology as a statistical tool for biotechnological applications: a critical appraisal," Biotechnology Journal , Vol. 3, pp. 510-523 (2008).

23. Ryu, K. S., Shaikh, K., Goluch, E., Fan, Z., and Liu, C., "Micro magnetic stir-bar mixer integrated with parylene microfluidic channels," Lab on a
Chip, Vol. 4, pp. 608-613 (2004).

24. Schwesinger, N., Frank, T., and Wurmus, H., "A modular microfluid system with an integrated micromixer," Journal of Micromechanics and Microengineering, Vol. 6, pp. 99-102 (1996).

25. Stroock, A. D., Dertinger, S. K. W., Ajdari, A., Mezic, I., Stone, H. A., and Whitesides, G. M., "Chaotic mixer for microchannels," Science, Vol. 295, pp. 647-651 (2002).

26. Trung, N., Nguyen, N., and Wu, Z., "Micromixer- a review," Journal of Micromechanics and Microengineering, Vol. 15, pp. 1-16 (2005).

27. Wang, C. T. and Hu, T. Y., "Biometric concept applied to micro-mixer design," The 4th International Green Energy Conference, October 20-22, Beijing, China (2008).

28. Wang, C. T., Hu, Y. C., and Hu, T. Y., "Biophysical micromixer design," Science, Vol. 9, pp. $5379-5389$ (2009).

29. Wong, S. H., Bryant, P., Ward, M., and Wharton, C., "Investigation of mixing in a cross-shaped micromixer with static mixing elements for reaction kinetics studies," Sensors and Actuators B: Chemical, Vol. 95, pp. 414-424 (2003)

30. Wong, S. H., Ward, M. C. L., and Wharton, C. W., "Micro T-mixer as a rapid mixing micromixer," Sensors and Actuators B: Chemical, Vol. 100, pp. 359-379 (2004)

31. Wu, Z. G. and Nguyen, N. T., "Convective-diffusive transport in parallel lamination micromixers," Microfluidics and Nanofluidics, Vol. 1, pp. 208-217 (2005)

32. Yang, Z., Matsumoto, S., Goto, H., Matsumoto, M., and Maeda, R., "Ultrasonic micromixer for microfluidic systems," Sensors and Actuators A: Physical, Vol. 93, pp. 266-272 (2001). 